\title{
Mycoplasma pneumoniae-Induced-Stevens Johnson Syndrome: Rare Occurrence in an Adult Patient
}

\author{
Samad Rasul, ${ }^{1}$ Faria Farhat, ${ }^{1}$ Yared Endailalu, ${ }^{2}$ Fatima Tabassum Khan, ${ }^{2}$ and Vishal Poddar ${ }^{3}$ \\ ${ }^{1}$ Division of Infectious Diseases, Department of Internal Medicine, Howard University Hospital, 2041 Georgia Avenue NW, \\ Washington, DC 20060, USA \\ ${ }^{2}$ Department of Internal Medicine, Howard University Hospital, 2041 Georgia Avenue NW, Washington, DC 20060, USA \\ ${ }^{3}$ Division of Pulmonary, Department of Internal Medicine, Howard University Hospital, 2041 Georgia Avenue NW, Washington, \\ DC 20060, USA
}

Correspondence should be addressed to Samad Rasul, dr.samad@gmail.com

Received 9 April 2012; Revised 2 July 2012; Accepted 22 July 2012

Academic Editor: Chin-Jung Wang

Copyright ( 2012 Samad Rasul et al. This is an open access article distributed under the Creative Commons Attribution License, which permits unrestricted use, distribution, and reproduction in any medium, provided the original work is properly cited.

\begin{abstract}
Stevens-Johnson syndrome (SJS) is an uncommon occurrence in Mycoplasma pneumoniae (M. pneumoniae) infection (1-5\%) and has been mainly reported in children and young adults. We present a case of SJS in a 32-year-old male induced by M. pneumoniae infection. This patient presented with fever, cough, and massive occupation of mucus membranes with swelling, erythema, and necrosis accompanied by a generalized cutaneous rash. He clinically responded after treatment with antibiotics and IVIG. SJS is usually a drug-induced condition; however, M. pneumoniae is the commonest infectious cause and should be considered in the differential diagnosis.
\end{abstract}

\section{Case Report}

A 32-year-old African American male presented to our hospital with a generalized skin rash for the past 3 days. He developed a productive cough, sore throat, and fever one week prior to developing this rash. He also noted rhinorrhea and pink eyes with visual blurring and was prescribed erythromycin eye ointment by a physician. The rash appeared one day using the ointment and was described as a nonpruritic, painless eruption of "bumps" starting on his back and rapidly spreading over his chest and hands. The rash progressed over 3 days and he experienced swelling of both eyelids along with blistering and crusting of his lips. He also experienced mild dysphagia and decided to come to the hospital for further evaluation. He admitted to being allergic to sulfa drugs; however, he had not received any antibiotics other than the erythromycin ointment. He denied any travel, animal exposure, or insect bites. He also denied having any sexual encounters in recent weeks. He had a history of occasional tobacco and marijuana usage, and his last use had been over 6 months ago.

On physical examination, he had a low-grade fever of 100.5 Fahrenheit, his pulse rate was 90 beats per minute, respiratory rate was 20 breaths per minute, blood pressure was $130 / 70 \mathrm{~mm}$ of $\mathrm{Hg}$, and he was saturating $90 \%$ on room air. His upper and lower eyelids were swollen bilaterally and he had obvious conjunctival erythema (Figure 1(a)). There were marked necrosis and mucosal sloughing involving his lips (Figure 1(b)). He had generalized macules and patches on his chest and back (Figures 1(c) and 1(d)), which to a lesser extent also involved his face, abdomen, and arms. The rash appeared in different stages of healing, and targetoid lesions were noted on selected sites most notably his palms (Figure 1(e)). His genitalia and lower extremities were not involved. On pulmonary examination, the right lower lung fields had fine end-inspiratory rales.

Pertinent laboratory results (with reference values in parenthesis) were as follows: serum creatinine $1.1 \mathrm{mg} / \mathrm{dL}$ $(0.7-1.4 \mathrm{mg} / \mathrm{dL})$, total bilirubin $0.9 \mathrm{mg} / \mathrm{dL}(0.2-1.2 \mathrm{mg} / \mathrm{dL})$, AST 99 IU/L (0-50 IU/L), ALT 31 IU/L (0-55 IU/L), alkaline phosphatase $37 \mathrm{IU} / \mathrm{L}$ (30-165 IU/L), albumin $3.1 \mathrm{grams} / \mathrm{dL}$ (3.2-5.5 grams/dL), total WBC count $11.6 \mathrm{cells} / \mathrm{mm}^{3}$ (3.2-10.6 cells $\left./ \mathrm{mm}^{3}\right)$, hemoglobin $13.3 \mathrm{grams} / \mathrm{dL}$ (12.115.9 grams $/ \mathrm{dL}$ ), and platelet count $228 \mathrm{cells} / \mathrm{mm}^{3}$ (175400 cells $/ \mathrm{mm}^{3}$ ). A urine toxicology screen was obtained and returned negative. His chest X-ray revealed a right lower 


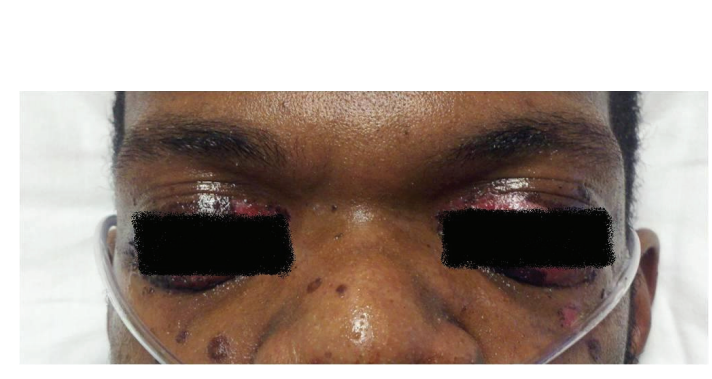

(a)

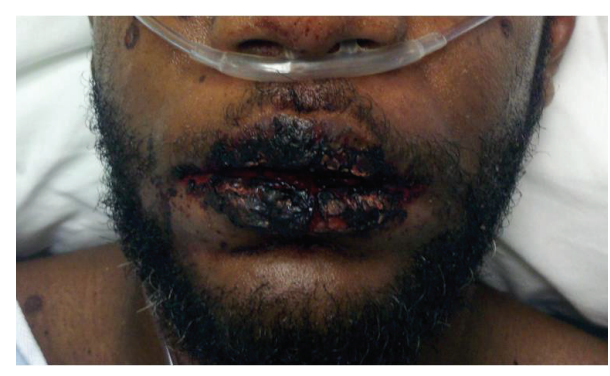

(b)

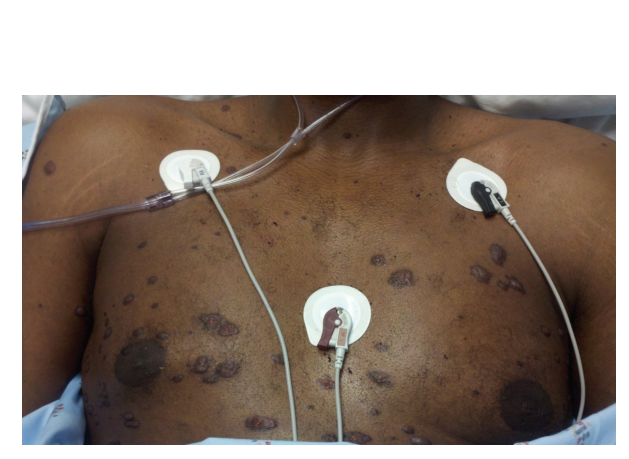

(c)

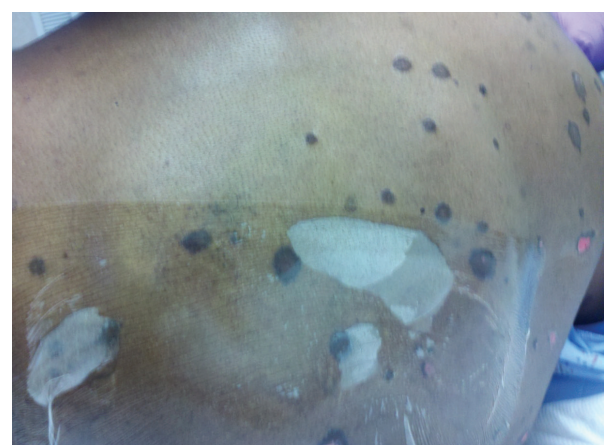

(d)

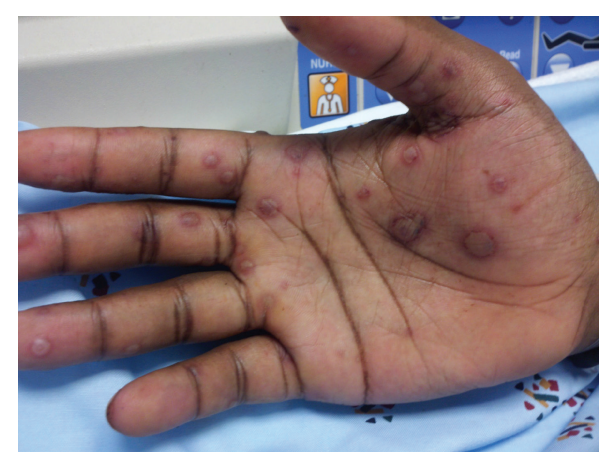

(e)

Figure 1: (a) Swelling and necrosis of eyelids with conjunctival erythema. (b) Severe necrosis of oral mucosa. (c) Macules and patches on chest. (d) Rash on back with areas of necrosis and denudation. (e) Targetoid lesions on right palm.

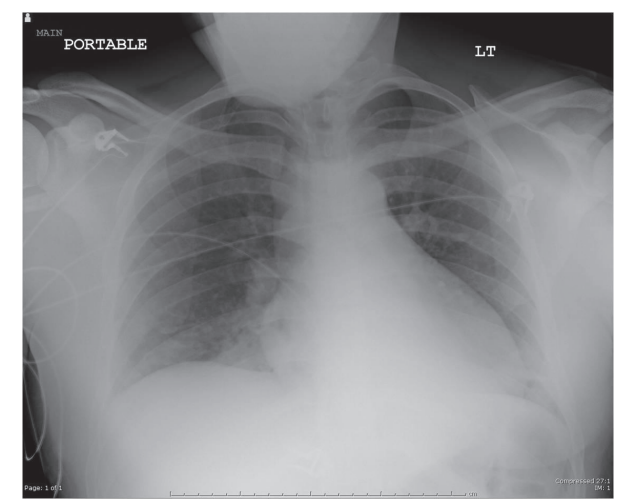

FIGURE 2: Right lower lung infiltrate on chest X-ray.

lobe infiltrate (Figure 2). Blood and urine cultures were negative. In view of characteristic skin rash with mucosal and conjunctival involvement, a diagnosis of Stevens-Johnson syndrome was considered. He was started on moxifloxacin and received methylprednisolone $80 \mathrm{mg}$ IV for a total of 4 doses with minimal response. He was then given $1 \mathrm{~g} / \mathrm{kg}$ intravenous immunoglobulin (IVIG) divided in to 3 doses, administered on consecutive days while continuing supportive care. Skin and mucus membrane lesions started to heal within 48 hours of IVIG.

A workup to determine the underlying etiology was pursued while supportive management was continued. Tests for syphilis, Epstein-Barr virus, Herpes simplex virus, Chlamydophila pneumoniae, hepatitis $\mathrm{B}$ and $\mathrm{C}$ viruses, influenza $\mathrm{A} / \mathrm{B}$ viruses, and human immunodeficiency virus (HIV) returned negative. A punch biopsy of skin from his left arm was performed and revealed subepidermal inflammation with necrotic infundibular epithelium and necrotic keratinocytes (Figures 3(a) and 3(b)) consistent with Stevens-Johnson syndrome. On day 5 of his hospital course, Mycoplasma pneumoniae IgM by enzyme immunoassay (EIA) returned at a level of $3010 \mathrm{U} / \mathrm{mL}(<770 \mathrm{U} / \mathrm{mL})$ with Mycoplasma pneumoniae $\operatorname{IgG}>5.00$ ISR $(\leq 0.90$ ISR negative, $0.91-1.09$ 


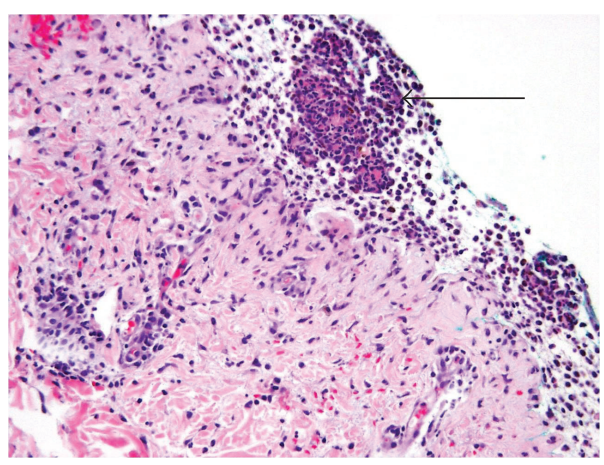

(a)

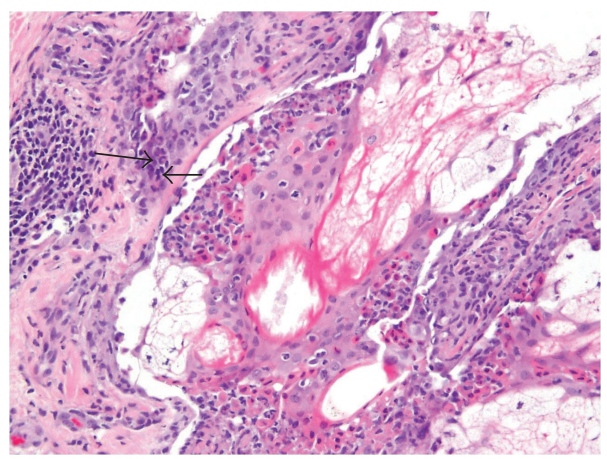

(b)

Figure 3: (a) Subepithelial abscess (arrow) on skin biopsy from left arm. (b) Necrotic infundibular epithelium with necrotic keratinocytes (arrows) on skin biopsy.

ISR equivocal, $\geq 1.10$ ISR positive). We thus concluded that this was a case of Stevens-Johnson syndrome secondary to Mycoplasma pneumoniae infection.

\section{Discussion}

Mycoplasma pneumoniae is one of the commonest causes of atypical pneumonia. 25-33\% of infected patients may show some cutaneous manifestations most notably exanthems, urticaria, and SJS [1]. Infection with M. pneumoniae may produce a variety of extrapulmonary manifestations, including neurological, hepatic, cardiac, and dermatological diseases before, during, or after pulmonary involvement [2]. SJS is a critical disease, characterized by high fever, extensive blistering lesions, mucosal involvement, and atypical targetlike skin lesions which often progress to toxic epidermal necrolysis (TEN) [2]. In addition to SJS, M. pneumoniae has also been noted to cause erythema multiforme (EM) and "atypical SJS" manifesting as severe mucositis without skin lesions [3]. The distinction between SJS and EM major is controversial; however mucous membrane involvement generally favors a diagnosis of SJS [3]. SJS usually evolves as an idiosyncratic reaction to medication (typically anticonvulsants or antibiotics), but $M$. pneumoniae has been cited as the most common infectious cause [3]. Many other infectious agents have been known to cause SJS, but none as commonly as M. pneumoniae [4].

M. pneumoniae-associated SJS most commonly affects children and young adults (mean age 15.3 years to 19 years) compared with drug associated SJS (mean age 31.4 years to 46.6 years) $[2,3]$. In younger patients, SJS may be preceded by symptoms of upper respiratory tract infection from 2 days to 2 weeks before the appearance of the rash [5]. Most adults generally develop symptoms and rash almost simultaneously, perhaps due to an immune reaction to previous exposure [2]. Oral lesions are present in $100 \%$ of cases, genital lesions in $75 \%$, and ocular lesions in 66\% [1]. M. pneumonia-induced SJS is associated with less severe complications and less internal organ involvement than those resulting from other causes $[2,3]$. Pneumonia is more commonly associated with drug-induced as opposed to M. pneumoniae-associated SJS [3].
Patients with M. pneumonia-associated SJS frequently receive antibiotics early during their illness before cutaneous involvement which may be a confounding factor in determining the precise etiology of SJS $[3,6]$. M. pneumoniae has occasionally been isolated from the involved skin of effected patients; however, these lesions are most commonly believed to result from immune complex-mediated vascular injury, autoimmune reaction, and cell-mediated immune response [6]. The diagnosis of SJS is clinical, and a detailed history should be obtained to identify and discontinue any potential causative drugs. A skin biopsy should be performed in all cases to exclude other conditions. M. pneumoniae work up should be considered in patients who present with SJS, particularly if there is no history of recent medication use [3].

The laboratory diagnosis of Mycoplasma infections can be obtained by culture, serology utilizing enzyme immunoassay (EIA), or complement fixation (CF) methods and polymerase chain reaction (PCR) based testing. Isolation of $M$. pneumoniae by culture is considered to be the gold standard of diagnosis, but requires 10-14 days and is not readable available [7]. Serology is most frequently used, and the most widely available methods are the CF and EIA tests [7]. The CF IgG antibody usually peaks 1-3 weeks after the onset of infection, and a fourfold increase in CF antibody titer is felt to be diagnostic [7]. EIA-basedtests that detect both $\operatorname{IgM}$ and $\operatorname{IgG}$ are more sensitive and specific than CF; however, the production of IgM antibodies may be inconsistent during the acute phase of infection, particularly in adults [7]. A high IgM antibody level by EIA method in our patient in the second week of illness strongly suggests that this was a case of acute Mycoplasma pneumoniae infection. PCR-based methods have gained popularity but are less sensitive compared to serological tests [8]. Some authors recommended using serology and PCR in parallel to diagnose Mycoplasma infection [8].

The management of $M$. pneumoniae-associated SJS includes supportive care with special attention to fluid and electrolyte balance $[3,9]$. Antibiotics have been used mostly for SJS due to M. pneumoniae infection or cases with secondary complications or sepsis [3]. The role of corticosteroids in the treatment of SJS is generally controversial due 
to concern for secondary infections [3]. However, recently published data from an observational study noted a possible beneficial effect in patients treated with corticosteroids [9]. IVIG (total dose of $3 \mathrm{~g} / \mathrm{kg}$ in divided doses over 3 days) may be considered in severe cases [3]. One observational study found significant benefit of using IVIG in children with druginduced SJS [10]. These findings have not been supported by others [11]. Evidence for IVIG use in adult patients is lacking. Sepsis or severe organ involvement has rarely been reported in M. pneumonia-associated SJS [3]. Younger patients are known to have a shorter course of hospital stay (<30 days) compared to adults [2]. The most common longterm complication is ocular involvement. Ophthalmology evaluation is recommended both at the time of diagnosis and prior to hospital discharge $[2,3]$.

We believe that this case is unique in a few aspects. (1) M. pneumoniae associated SJS is more common in children and young adults $<21$ years of age and is unusual in patients above this age range. (2) Our patient developed URI and conjunctival symptoms 1 week before skin lesions and mucositis, which is an atypical lag period in adults. (3) Our patient had clinical and radiological evidence of pneumonia, which is less common in M pneumonia-associated SJS. (4) Our patient had high levels of Mycoplasma pneumoniae IgM antibodies which is not common for primary infections or reexposure in adults. Based on these findings, we suggest that M. pneumoniae infections can have an atypical presentation in adults and should be considered in the differential diagnosis of SJS in the appropriate clinical setting.

\section{Disclosure}

The authors solemnly declare that they have no financial disclosures. This manuscript has not been published nor is under consideration for publication elsewhere. All coauthors have agreed to the submission of this publication.

\section{References}

[1] P. C. Schalock and J. G. H. Dinulos, "Mycoplasma pneumoniae-induced cutaneous disease," International Journal of Dermatology, vol. 48, no. 7, pp. 673-681, 2009.

[2] Y. Kunimi, Y. Hirata, M. Aihara et al., "Statistical analysis of Stevens-Johnson syndrome caused by Mycoplasma pneumonia infection in Japan," Allergology International, vol. 60, pp. 525532,2011

[3] D. A. Wetter and M. J. Camilleri, "Clinical, etiologic, and histopathologic features of Stevens-Johnson syndrome during an 8-year period at Mayo Clinic," Mayo Clinic Proceedings, vol. 85, no. 2, pp. 131-138, 2010.

[4] M. Walicka, M. Majsterek, A. Rakowska et al., "Mycoplasma pneumoniae-induced pneumonia with Stevens-Johnson syndrome of acute atypical course," Polskie Archiwum Medycyny Wewnetrznej, vol. 118, no. 7-8, pp. 449-453, 2008.

[5] V. Tsai and J. Oman, "Stevens-Johnson syndrome after $M y$ coplasma pneumoniae infection," The Journal of Emergency Medicine, vol. 40, no. 3, pp. 324-327, 2011.

[6] J. D. Cherry, "Anemia and mucocutaneous lesions due to Mycoplasma pneumoniae infections," Clinical Infectious Diseases, vol. 17, supplement 1, pp. S47-S51, 1993.
[7] M. R. Hammerschlag, "Mycoplasma pneumoniae infections," Current Opinion in Infectious Diseases, vol. 14, no. 2, pp. 181186, 2001.

[8] M. A. Martínez, M. Ruiz, E. Zunino, V. Luchsinger, and L. F. Avendaño, "Detection of Mycoplasma pneumoniae in adult community-acquired pneumonia by PCR and serology," Journal of Medical Microbiology, vol. 57, no. 12, pp. 1491-1495, 2008.

[9] J. Schneck, J. P. Fagot, P. Sekula, B. Sassolas, J. C. Roujeau, and M. Mockenhaupt, "Effects of treatments on the mortality of Stevens-Johnson syndrome and toxic epidermal necrolysis: a retrospective study on patients included in the prospective EuroSCAR Study," Journal of the American Academy of Dermatology, vol. 58, no. 1, pp. 33-40, 2008.

[10] D. W. Metry, P. Jung, and M. L. Levy, "Use of intravenous immunoglobulin in children with Stevens-Johnson syndrome and toxic epidermal necrolysis: seven cases and review of the literature," Pediatrics, vol. 112, no. 6, pp. 1430-1436, 2003.

[11] N. Bachot, J. Revuz, and J. C. Roujeau, "Intravenous immunoglobulin treatment for Stevens-Johnson syndrome and toxic epidermal necrolysis: a prospective noncomparative study showing no benefit on mortality or progression," Archives of Dermatology, vol. 139, no. 1, pp. 33-36, 2003. 


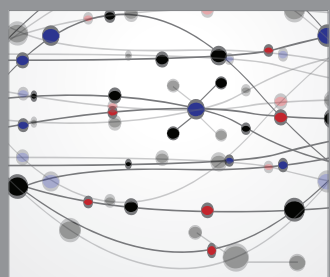

The Scientific World Journal
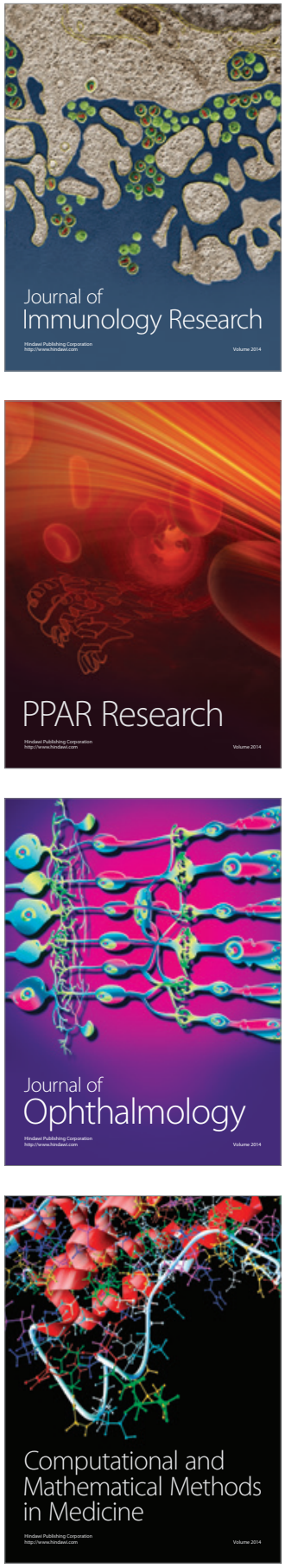

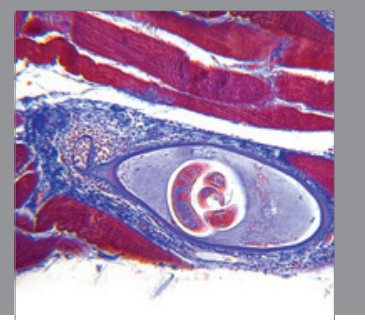

Gastroenterology

Research and Practice
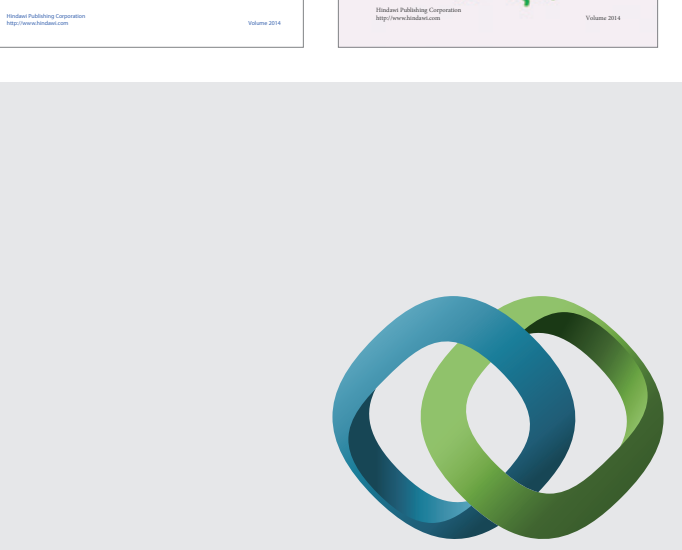

\section{Hindawi}

Submit your manuscripts at

http://www.hindawi.com
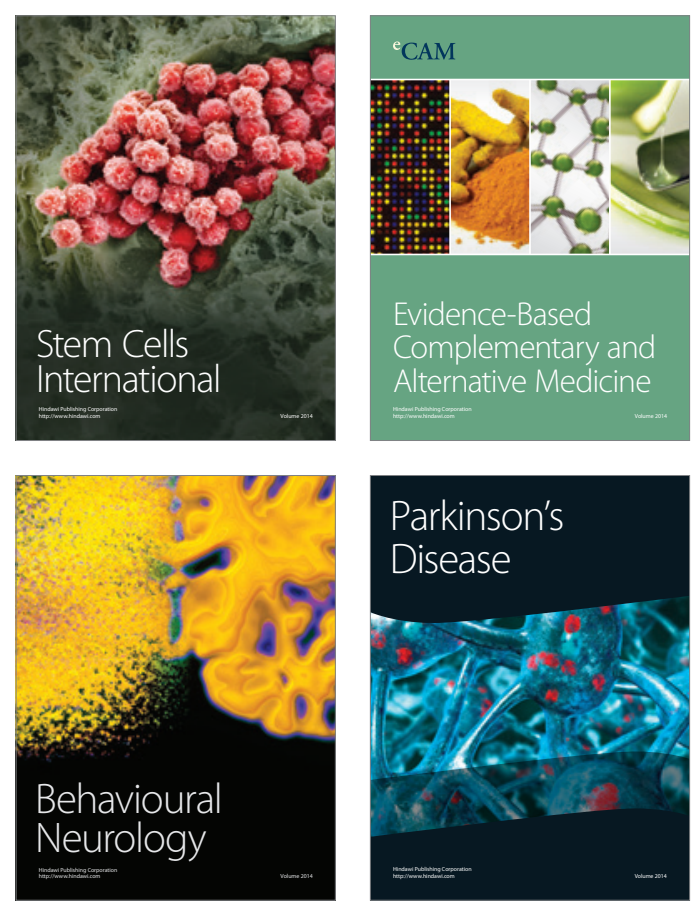

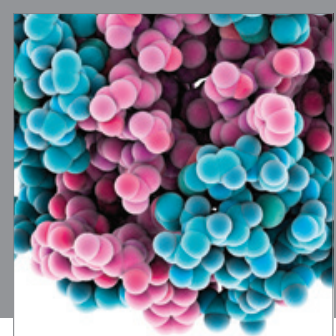

Journal of
Diabetes Research

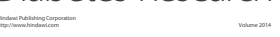

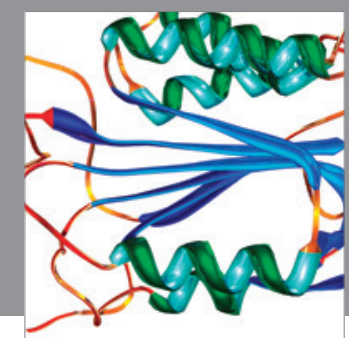

Disease Markers
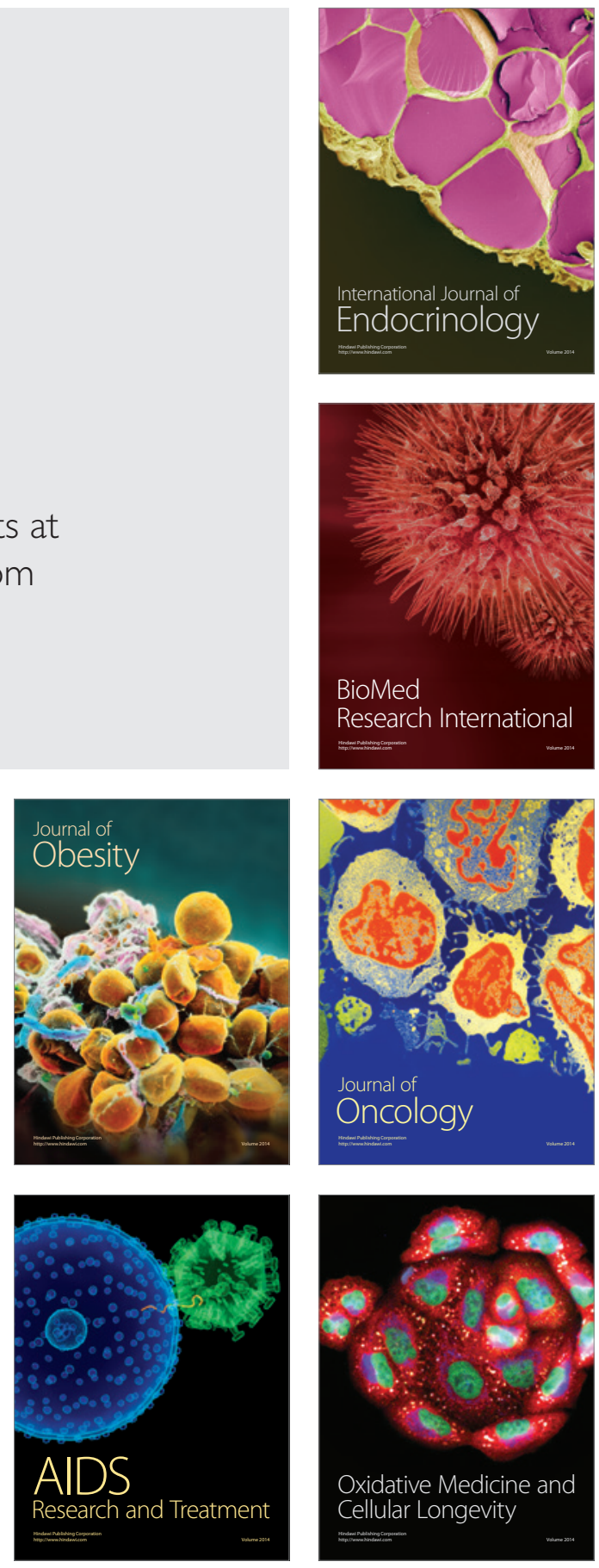